\title{
Optimal power control and coverage management in two-tier femtocell networks
}

\author{
Georgios Aristomenopoulos ${ }^{*}$, Timotheos Kastrinogiannis, Stamatina Lamprinakou and Symeon Papavassiliou
}

\begin{abstract}
In this article, we jointly consider the problem of efficient power control and coverage (PCC) management over an integrated two-tier macrocell/femtocell network towards maximizing the expected throughput of the system subject to appropriate power constraints, under the existence of both co-tier and cross-tier interferences. Optimal network design amounts to joint optimization of users' allocated power levels and cell's maximum aggregated downlink transmitted power, i.e., coverage area management. This problem is inherently difficult because it is in fact a non-convex optimization problem. A novel approach to address the latter is performed that entails a suitable transformation, which allows the use of convex optimization and also forms the basis for the design of a distributed PCC algorithm via performing two-level primal-dual decomposition. PCC algorithm's convergence to optimality is established. We demonstrate that for realistic macrocell/femtocell deployment scenarios, overall system throughput increase up to approximately 50\% can be achieved while guaranteeing $70 \%$ of power savings.
\end{abstract}

Keywords: Two-tier femtocell networks, Power control, Coverage management' non-convex optimization

\section{Introduction}

In recent years, the increasing demand for efficient indoor coverage coupled with the need for high data rates and quality of service (QoS) has highlighted the inflexibilities of the so-called traditional macrocell layer of a cellular network, calling for new indoor coverage/ capacity management solutions. In response to this problem, the use of femtocell access points or home base stations [1] has been proposed. Femtocell networks are created by low-power, low-cost, user-deployed base stations that are able of providing high-quality cellular service in residential or enterprise environments while operating in licensed spectrum.

As femtocells share spectrum with macrocell-network, efficient cross-tier interference mitigation between femtoand macrocells is essential to facilitate both coverage and capacity enhancements. Nevertheless, efficient two-tier power control and resource allocation faces several additional design challenges compared to their single-tier cellular counterparts. First, co-tier interference, in terms of intra- and inter-cell interference (e.g., between neighboring

\footnotetext{
* Correspondence: aristome@netmode.ntua.gr

Institute of Communications and Computer Systems (ICCS), School of

Electrical and Computer Engineering, National Technical University of Athens, Athens 15780, Greece
}

femtocells), along with cross-tier interference need to be jointly considered in the computation of users' transmission environment (i.e., the achieved signal-to-interference plus noise ratio, SINR), resulting in non-convex power control optimization settings. Second, the network operator agnostic deployment of femtocells demands their ability to dynamically sense the radio environment and adjust their size accordingly (via tuning their transmission power level) towards minimizing the coverage area leak into neighboring cells [2]. Therefore, along with power control, efficient, distributed, and dynamic coverage management $(\mathrm{CM})$ need to be considered.

This study deals with optimal joint power control and coverage (PCC) management in the presence of co-tier and cross-tier interferences in two-tier code-division multiple-access (CDMA) macrocell/femtocell wireless networks. The goal is to find the optimal operation point that maximizes network-wide throughput performance, in terms of users' downlink transmission power and cells' maximum cumulative power assigned to users (i.e., cells' coverage size). The large number of variables involved and the non-convexity of the optimization problem associated to the latter goal reveal the difficulty in obtaining such an operation point. 


\section{Limitations of existing literature work}

Such issues are typically addressed in the literature through the isolation and individual treatment of specific goals of the overall two-tier network optimization problem, leading to fewer degrees of freedom. To that end, for treating femtocells' CM problem only, a large variety of dynamic cell sizing schemes have been developed towards improving overall system's capacity [3-10], when compared to that of fixed cell sizing schemes. This is achieved by the dynamic adaptation of femtocells' maximum transmission power [3-5] or pilot signal power [7-9] in a step-wise heuristic manner, while aiming at one of the following goals: (a) assure an upper bound on femtocell user's SINR [3], (b) minimize femtocells' coverage leakage probability (i.e., the probability that a femtocell user's SINR is larger than a threshold, towards minimizing femtocells' coverage area leak into an outdoor macrocell) [5], (c) minimize the amount of handovers between the two tiers [7], or (d) minimize power consumption [9].

Furthermore, a vast number of two-tier power control and interference mitigation problems have been proposed and studied towards serving a variety of alternative goals, i.e., from overall system interference minimization [11-13] to overall system throughput optimization [14], and from overall power minimization under SINR [15], QoS [16], and power [17] constraints, to integrated system outage probability minimization [18] and utilitybased optimization $[19,20]$.

Recently in [21], a distributed and self-organizing femtocell management architecture has been proposed to jointly mitigate two-tier network interference. The proposed architecture consists of three control loops to determine (1) the maximum transmit power of femtocell users, (2) a target SINRs of femtocell users to reach a Nash equilibrium, and (3) instantaneous transmit power of femtocell users to achieve that target SINRs. Although both power control and CM are considered in [21], their correlation is limited to a specific periodic information exchange between the control loops that enable the latter mechanisms, leading to sub-optimal heurist solutions.

\section{Article's contributions and methodology}

The previous analysis highlights the lack of an efficient theoretically sound framework to jointly incorporate PCC management over a macrocell/femtocell system. This would provide enhanced flexibility on combining both mechanisms' interference mitigation attributes towards optimizing overall users' performance in the two-tier system. This article develops an optimization framework that fills the above gap.

To that end, we initially formulate the two-tier joint PCC management non-convex optimization problem (P1) and then, transform it into an equivalent convex one. The latter is further treated via a two-level primaldual decomposition, as illustrated in Figure 1, leading to distributed PCC mechanism that provably obtains optimality. At the lower decomposition level, intra-cell power control (IPC) problems derive users' transmit power vectors achieving optimal intra-cell resource allocation. At a higher level, a CM problem is responsible for dynamically determining optimal cells' maximum cumulative transmission power vector (i.e., coverage area) towards network-wide performance optimality. CM is accomplished by utilizing a subgradient method which exploits cells' load/congestion prices (i.e., the Lagrange multipliers of ICPs' dual problems), that turn out to be the optimal "twotier layering prices", leading to a distributed low-signaling algorithmic solution.

In brief, the proposed PCC management framework and corresponding PCC algorithm claims the following design attributes.

- Explicit CM. In the proposed optimization framework, the maximum transmission power of each femtocell is considered as an optimization variable (properly constrained by a lower and upper bound) incorporated to the overall power control optimization problem. This enables explicit femtocells' CM towards network-wide throughput optimality.

- Network-wide optimality. Due to cross-tier and co-tier interferences, the initial PCC management problems is non-convex and thus, hard to solve, especially in a distributed manner. To that end, a novel approach to address that latter is performed that entails a suitable transformation, which allows the use of convex optimization and also forms the basis for the design of a distributed PCC algorithm via performing two-level primal-dual decomposition.

- Distributed implementation and convergence analysis. The resulting architectural framework is enabled via a distributed, low-complexity subgradient algorithm. This attribute reassures low excess signaling overhead and favors scalability. PCC algorithm's convergence to the network-wide global optimal is proven, while its convergence speed and imposed signaling overhead are discussed.

The rest of the article is organized as follows. "System model" section describes the adopted system model, while in "Problem formulation and transformations" section the problem formulation and its transformation are detailed. In "PCC management for a two-tier femtocell/macrocell system" section, problem's primal-dual decomposition and its solution via a subgradient method are provided, while in "Distributed PCC algorithm" section PCC algorithm's optimality and convergence attributes are 


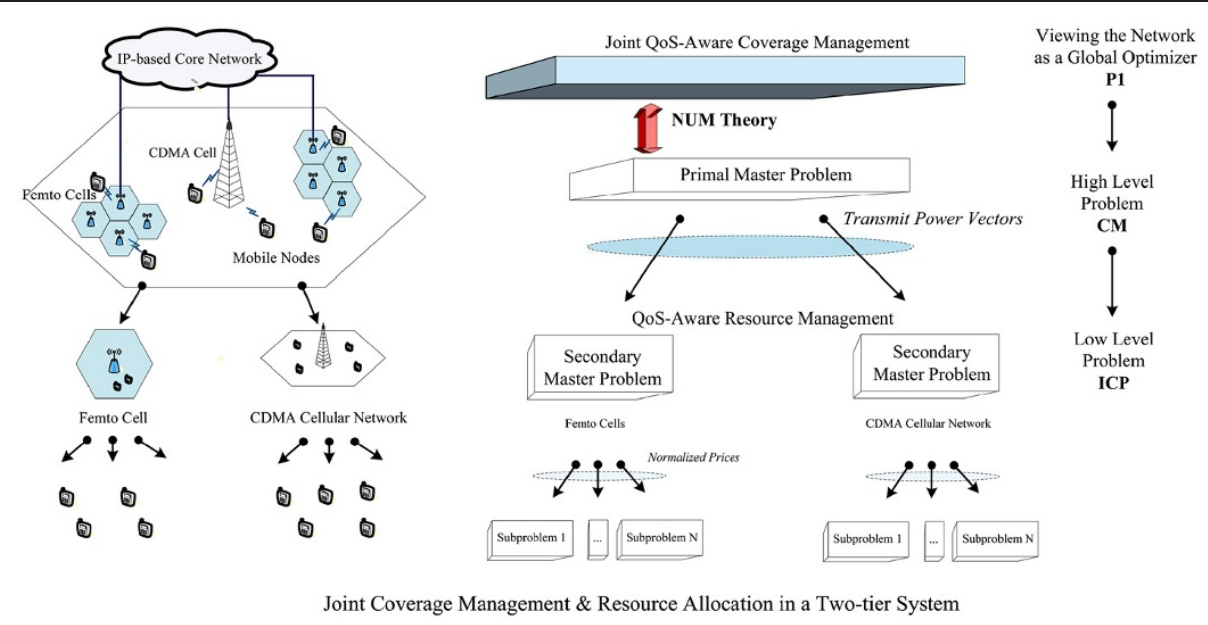

Figure 1 Two-tier femtocell/macrocell PCC management as a global optimizer via NUM decomposition.

discussed. Finally, in "Numerical results" section extensive numerical results, demonstrating the efficacy of the proposed approach, are presented and discussed, while "Concluding remarks and future work" section concludes this article.

\section{System model}

In this article, we consider a two-tier femtocell/macrocell network with a set $C$ of $|C|$ cells. The system consists of a single central macrocell $C_{0}$ and $|C|-1$ underlay cochannel femtocells $C_{c}$, where $c=1, \ldots,|C|-1$. We further consider a set $J$ of $|J|$ active mobile users located in the above area. On every occasion, a user $j \in J$ can be attached to only one cell $c \in C$. This study assumes closed access (CA) [1], which means only licensed home users, within radio range, can communicate with their own femtocell. We denote as $S_{c}$ the set of $\left|S_{c}\right|$ users served by base station $c$ thus, $J=S_{0} \times \cdots \times S_{|C|-1}$ is a Cartesian product.

The system is assumed to be time-slotted, while orthogonal CDMA downlink is assumed in each slot. All cells use the same frequency channel [i.e., share the same spreading bandwidth $W(\mathrm{~Hz})]$. Therefore, to exploit multi-user diversity, at each time-slot active mobile users' downlink transmission power control and cells' coverage (PCC) management algorithm is executed.

The channel between cells' BSs/APs transmitters and mobile nodes' receivers is modeled as an additive white Gaussian noise multi-access channel. Let us denote by $G_{c, j}$ the path gain between cell $c$ and user $j$, reflecting the long-time behavior of the channel gain. Fast-fading can be considered via smoothing out by appropriate averaging [22]. We focus on one time-slot assuming that the path gain, background noise, and inter-cell interference for each mobile do not change during this time-slot $[23,24]$. Hence, the notion of time is omitted in the definition of the variables introduced in the rest of the article.
Within the duration of a timeslot, each mobile $j \in S_{c}$ communicates with its corresponding cell's base station and receives data at a certain downlink transmission power $P_{c, j}$. Furthermore, considering cell's $c$ overall downlink transmission, there exists an intra-cell cumulative power, $P_{c \text {,var }}$ which can be allocated to the attached mobiles (i.e., the sum of the power allocated to each mobile cannot exceed this value); therefore, $\sum_{\mathrm{j} \in \mathrm{S}_{\mathrm{c}}} P_{c, \mathrm{j}} \leq P_{c, \mathrm{var}}$. Furthermore, we denote by $\bar{P}_{c}=\left(P_{c, 1}, \ldots, P_{c,\left|S_{c}\right|}\right)$ cell's $c$ power allocation vector and by $\bar{P}=\left(\bar{P}_{0}, \bar{P}_{1}, \ldots, \bar{P}_{|C|-1}\right)$ the two-tier system power vector. In this study, to enable efficient $\mathrm{CM}$ and thus interference mitigation, the cumulative downlink transmission power for each cell $c, P_{c \text {,var }}$ is not fixed, but can be dynamically tuned. Of course, the latter is both upper and lower bounded by $P_{c}^{\mathrm{Min}} \leq P_{c \text {,var }} \leq P_{c}^{\mathrm{Max}} \forall c \in C$, due to cell's physical limitations. We define as $\gamma_{c, j}$ the SINR at user $j$ from BS $c$ as follows.

$$
\gamma_{c, j}=\frac{G_{c, j} P_{c, j}}{G_{c, j} \sum_{j^{\prime} \neq j}^{j^{\prime} \in S_{c}} P_{c, j^{\prime}}+\sum_{c^{\prime} \neq c}^{c^{\prime} \in C} G_{c^{\prime}, j}\left(\sum_{k \in S_{c^{\prime}}} P_{c^{\prime}, k}\right)+n_{0}}
$$

where the denominator incorporates intra-cell, co- and cross-tier interference, and background noise, respectively.

Each user $j$ is associated with a generic monotonic strictly concave utility function $U_{j}\left(\gamma_{c, j}\right)$ of the achieved SINR, expressing the data rates attainable on the wireless links. The goal is to maximize the sum of two-tier users' utilities under proper constraints, i.e., optimize system's overall throughput efficacy. Therefore, towards incorporating a large family of modulations, $U_{j}$ is expressed as a global, nonlinear function of the transmit power vector and channel conditions [25], i.e.,

$$
U_{j}\left(\gamma_{c, j}(\bar{P})\right)=\frac{1}{T} \log _{2}\left(1+K \gamma_{c, j}(\bar{P})\right)
$$


where constant $T$ is the symbol period, which will be assumed to be one unit without loss of generality, and constant $K=\left(-\phi_{1}\right) / \log \left(\phi_{2} \mathrm{BER}\right)$, where $\phi_{1}$ and $\phi_{2}$ are constants depending on the modulation, and BER is the required bit error rate [26]. Finally, assuming high-SINR regime [i.e., that $K \gamma_{c, j}(\bar{P})$ is much larger than 1], which is commonly adopted in power control $[27,28], U_{j}$ can be approximated by

$$
U_{j}\left(\gamma_{c, j}(\bar{P})\right)=\log \left(\gamma_{c, j}(\bar{P})\right)
$$

This approximation is reasonable either when the signal level is much higher than the interference level (as in the case of a femtocell system, due to the proximity of the femtocell user's at their base stations) or when the spreading gain is large. In (2), for simplicity in the presentation $K$ has been incorporated and absorbed into $G_{c, j}$. Finally, it should be highlighted that while $U_{j}$ is a nonlinear non-concave function of $\bar{P}$, it can be converted into a concave function through an appropriate log transformation, leading to a critical convexity property that establishes global optimality (see "PCC management for a two-tier femtocell/macrocell system" section).

\section{Problem formulation and transformations}

Under the setting described in the previous section, the problem of joint power allocation and CM in the two-tier femtocell/macrocell system comprising of $|C|$ cells and $|J|$ users amounts to devising cells' optimal transmission power $P_{c \text {, var }} \forall c \in C$ and the corresponding users' optimal power allocation vector $\bar{P}$ that will maximize the total expected system throughput. Considering both optimization variables the optimization problem can be defined as

$$
\begin{aligned}
& \text { (P1) } \operatorname{maximize}_{\bar{P}, \bar{P}_{\text {var }}} \quad \sum_{j \in J} U_{j}\left(\gamma_{c, j}(\bar{P})\right) \\
& \text { s.t. } \quad \sum_{j \in S_{c}} P_{c, j} \leq \mathrm{P}_{c, \text { var }} \quad \forall c \in C \\
& P_{c}^{\text {Min }} \leq \mathrm{P}_{c, \text { var }} \leq \mathrm{P}_{c}^{\text {Max }} \quad \forall c \in C
\end{aligned}
$$

where the computation of both vectors $\bar{P}=\left(\bar{P}_{0}, \bar{P}_{1}, \ldots, \bar{P}_{|C|-1}\right)$ and $\bar{P}_{\text {var }}=\left(P_{0, \text { var }}, P_{1, \text { var }}, \ldots, P_{|C|-1 \text {. var }}\right)$ enables the integration of system's CM into overall system's power control and interference management optimization problem. Therefore, our goal is to jointly maximize the total system utility (e.g., the total expected system throughput) and to obtain the optimal cell's coverage, in terms of maximum intra-cell transmission power, with constraints on cells' cumulative transmission power to the attached users (i.e., constraint (4)) and base stations' maximum intra-cell transmission power due to physical limitations (i.e., constraint (5)).

\section{A necessary condition to optimality (first problem transformation)}

To solve problem (P1) in an efficient and distributed manner via optimization decomposition, we first need to overcome the complexity imposed by the coupling of the optimization variables in problem's objective function, due to the nature of the two-tier SINR in (1). To this end, we provide the following proposition.

Proposition 1: A necessary condition to achieve maximum two-tier overall system utility is that all base stations $c \in C$ must assign all their resources to their attached users and thus transmit at their maximum intra-cell available cumulative transmission power $P_{c, \text { vav }}$ for any given feasible $\bar{P}_{\text {var }}$ (i.e., when equalities in (4) hold).

Proof: See Appendix.

Proposition 1 allows to consider that $\sum_{j \in S_{c}} P_{c, j}=$ $P_{c \text {, var }} \forall c \in C$. Hence, we can rewrite a user's SINR [Equation (1)] as a function of only (i) his allocated power and (ii) the corresponding system's maximum intra-cell cumulative power limit vector $\bar{P}_{\text {var, }}$ as shown bellow.

$$
\begin{aligned}
\gamma_{c, j}(\bar{P}) & =\frac{G_{c, j} P_{c, j}}{G_{c, j}\left(P_{c, \mathrm{var}}-P_{c, j}\right)+\sum_{c^{\prime} \neq c}^{c^{\prime} \in C} G_{c^{\prime}, j} P_{c^{\prime}, \mathrm{var}}+n_{0}} \\
& =\frac{G_{c, j} P_{c, j}}{\sum_{c^{\prime} \in C} G_{c^{\prime}, j} P_{c^{\prime}, \mathrm{var}}+n_{0}-G_{c, j} P_{c, j}} \\
& =\frac{P_{c, j}}{\sum_{c^{\prime} \in C} \frac{G_{c^{\prime}, j}}{G_{c, j}} P_{c^{\prime}, \mathrm{var}}+\frac{n_{0}}{G_{c, j}}-P_{c, j}} \\
& =\frac{P_{c, j}}{I_{c, j}\left(\bar{P}_{\mathrm{var}}\right)-P_{c, j}} \triangleq \gamma_{c, j}\left(P_{c, j}, \bar{P}_{\mathrm{var}}\right)
\end{aligned}
$$

where $I_{c, j}\left(\bar{P}_{\text {var }}\right)$ intuitively reflects the transmission environment between mobile user $j$ and base station $c$, for a given macrocell/femtocells CM configuration. Hence, we can rewrite the expected throughput of mobile $j$ defined in (2) as $U_{j}\left(\gamma_{c, j}(\bar{P})\right)=U_{j}\left(\gamma_{c, j}\left(P_{c, j}, \bar{P}_{\text {var }}\right)\right)$. In line with the above, the joint power allocation and CM problem (P1) in the two-tier femtocell/macrocell system can be rewritten into the following way summating over all connected users $j \in S_{c}$ of each cell $c \in C$ in the system. We refer to this as (P2):

$$
\begin{aligned}
& \text { (P2) } \underset{\bar{P}, \bar{P}_{\mathrm{var}}}{\operatorname{maximize}} \sum_{c \in C} \sum_{j \in S_{c}} U_{j}\left(\gamma_{c, j}\left(P_{c, j}, \bar{P}_{\mathrm{var}}\right)\right) \\
& \text { s.t. } \quad \sum_{j \in S_{c}} P_{c, j} \leq \mathrm{P}_{c, \text { var }} \quad \forall c \in C \\
& P_{c}^{M i n} \leq \mathrm{P}_{c, \mathrm{var}} \leq \mathrm{P}_{c}^{\text {Max }} \quad \forall c \in C
\end{aligned}
$$

Therefore, our goal in this article is to establish a joint PCC management algorithm that distributively attains the jointly globally optimal solution $\left(\left(\bar{P}_{*}, \bar{P}_{\text {var }} *\right)\right)$ to 
problem (P2) and thus, the equivalent (P1). However, we first need to overcome two major challenges imposed by the global dependencies in (P2), that is

- Non-convexity. Although problem's (P2) constraints are convex and preserve a decoupled attribute with respect to system cells, variables $\bar{P}$ and $\bar{P}_{\text {var }}$ are globally coupled across the two-tier system, as reflected in the range of summations in the objective function (7), due to (6). As a consequence, problem (P2) is generally non-convex and hence challenging to solve.

Treatment: Via a log transformation $[29,30]$, we convert (P2) into an equivalent convex optimization problem (P3) (see "Pursuing convexity (second problem transformation)" section). This enables the proposed PCC algorithm with the following desirable properties: (a) global convergence to optimality $\left(\bar{P} *, \bar{P}_{\text {var }} *\right)$; (b) elegant tradeoff between complexity and performance, and (c) geometric rate of convergence.

- Deriving a distributed solution. The nonlinear optimization problem (P2) may be solved by centralized computation using the interior-point method for convex optimization [31], i.e., geometric programming [32]. However, in the context of wireless femtocell networks a distributive algorithm is required towards minimizing imposed signaling overhead and improving accuracy.

Treatment: The convex equivalent formulation of problem (P2) [that is problem (P3)] is further solved via two-level primal-dual optimization decomposition (see "PCC management for a twotier femtocell/macrocell system" section). Hence, two distributed algorithms for jointly attaining optimal (a) CM and (b) intra-cell's power allocation are proposed and analyzed.

\section{Pursuing convexity (second problem transformation)}

In accordance to the previous paragraph, in order to address problem's (P2) non-convexity, we apply the following one-to-one change of variables $P_{c, i}=e^{p_{c, i}}$ and $P_{c, \text { var }}=e^{p_{c} \text {, var }} \quad \forall j \in J, \forall c \in C$ in the original problem. After simple mathematical manipulations, (P2) can be equivalently written as follows.

$$
\begin{aligned}
& \text { (P2) } \underset{\bar{P}, \bar{P}_{\mathrm{var}}}{\operatorname{maximize}} \sum_{c \in C} \sum_{j \in S_{c}} U_{j}\left(\gamma_{c, j}\left(P_{c, j}, \bar{P}_{\mathrm{var}}\right)\right) \\
& \text { s.t. } \quad \sum_{j \in S_{c}} \exp \left(p_{c, j}-p_{c, \mathrm{var}}\right) \leq 1 \quad \forall c \in C \\
& P_{c}^{\text {Min }} \exp \left(-p_{c, \mathrm{var}}\right) \leq 1 \quad \forall c \in C \\
& \left(P_{c}^{\text {Max }}\right)^{-1} \exp \left(p_{c, \mathrm{var}}\right) \leq 1 \quad \forall c \in C
\end{aligned}
$$

The transformed constraints are convex in $\bar{p}=\left(\bar{p}_{0}, \bar{p}_{1}, \ldots\right.$, $\left.\bar{p}_{|C|-1}\right)$ and $\bar{p}_{\text {var }}=\left(p_{0, \mathrm{var}}, p_{1, \mathrm{var}}, \ldots, p_{|C|-1, \mathrm{var}}\right)$ since all left-hand sides are compositions of non-negative sum of exponentials, which are convex functions with affine mappings as shown in [28]. What remains to be established is the convexity of the objective function (9) in $\bar{p}$ and $\bar{p}_{\text {var }}$. From (9) we have,

$$
\begin{aligned}
& \sum_{c \in C} \sum_{j \in S_{c}} U_{j}\left(\gamma_{c, j}\left(p_{c, j}, \bar{p}_{\text {var }}\right)\right)= \\
& \sum_{c \in C} \sum_{j \in S_{c}} \log \frac{G_{c, j} \exp \left(p_{c, j}\right)}{\sum_{c^{\prime} \in C} G_{c^{\prime}, j} \exp \left(p_{c^{\prime}, \operatorname{var}}\right)-G_{c, j} \exp \left(p_{c, j}\right)+n_{0}}= \\
& \sum_{c \in C} \sum_{j \in S_{c}}\left[\log \left(\exp \left(p_{c, j}+\ln \left(G_{c, j}\right)\right)\right)\right. \\
& -\log \left(\sum_{c^{\prime} \in C} \exp \left(p_{c^{\prime}, \operatorname{var}}+\ln G_{c^{\prime}, j}\right)\right. \\
& \left.\left.-\exp \left(p_{c, j}+\ln \left(G_{c, j}\right)\right)+\exp \left(\ln n_{0}\right)\right)\right]
\end{aligned}
$$

The first term in the square bracket is linear in $\bar{p}$ and $\bar{p}_{\text {var }}$, since it is linear in $p_{c, j}$. The second term is a logarithmic summation of exponentials of linear functions of $\bar{p}$ and $\bar{p}_{\text {var }}$, which is concave in the latter domain, as proven in $[25,29]$. Finally, since the objective function of (P3) is a sum of concave terms, we conclude that (P3) is a convex optimization problem.

\section{PCC management for a two-tier femtocell/ macrocell system}

The convex optimization problem (P3) has two features which facilitate a distributed solution. First, the objective in (10) is a sum of $|C|$ intra-cell $\left|S_{c}\right|$ users' utilities summations, that depend only on the variables $\bar{p}_{c}$ and $\bar{p}_{\text {var. }}$. Second, the constraints (11)-(13) also depend only on $\bar{p}_{c}$ and $\bar{p}_{\text {var }}$. Based on the latter features, in this section an algorithm based on Lagrangian techniques for obtaining the solution of problem (P1) by solving problem (P3) is developed (i.e., deriving $\bar{p}_{c}{ }_{c}$ and $\bar{p}^{*}$ var).

Applying primal-dual decomposition [33] on (P3), as depicted in Figure 1, results in

I. $|C|-1$ independent IPC sub-problems. Considering first primal decomposition of (P3) by fixing $\bar{p}_{\text {var }}$ (i.e., for a given cells' coverage assignment) problem (P3) breaks in $|C|-1$ independent IPC sub-problems, each one responsible for computing the optimal intracell power vector $\bar{p}^{*}{ }_{c}$ for a given $\bar{p}_{\text {var }}$ allocation. IPC' solution is provided in "Treating IPC" section.

II. A master problem, responsible for updating the value of $\bar{p}_{\text {var }}$ towards obtaining $\bar{p}^{*}$ var. This master problem eventually performs CM [therefore denoted as problem (CM)] via controlling the maximum intra-cell cumulative power vector, and thus the coverage range, of each cell in the integrated system. To solve (CM), we use a subgradient method exploiting the information of 
IPCs' Lagrange multipliers $\lambda_{c}{ }^{*} \forall c \in C$ associated to the constraint (10), as detailed in "Deriving the lagrange multiplier of an (IPCs)" section.

It is important to note that since (P3) is a convex optimization problem, both master problem (CM) and sub-problems (IPCs) are also convex optimization problems [34]. In the following, we provide the solutions of IPCs and CM, respectively.

\section{Treating IPC}

By fixing $\bar{P}_{\text {var }}$, problem (P3) becomes separable and transforms to $|C|-1$ independent IPC sub-problems individually solvable by each cell $c$ since, (a) the objective function is a composition of $|C|-1$ independent summations that depend only on $\bar{p}_{c} \forall c \in C$, (b) constraints set is also separable in $C$, and (c) $J=S_{0} \times S_{1} \times \cdots \times S_{|C|-1}$ is a Cartesian product, since $\mathrm{CA}$ is assumed. Therefore, we define the IPC problem as

$$
\begin{aligned}
& \left(\mathrm{IPC}_{c}\right) \underset{\bar{p}_{c}}{\operatorname{maximize}} \mathrm{U}_{c}\left(\bar{p}_{c}\right) \equiv \sum_{j \in S_{c}} U_{j}\left(\gamma_{c, j}\left(p_{c, j}\right)\right) \\
& \text { s.t. } \quad \sum_{j \in S_{c}} \exp \left(p_{c, j}-p_{c, \mathrm{var}}\right) \leq 1
\end{aligned}
$$

Problem $\left(\mathrm{IPC}_{c}\right)$ obtains optimal intra-cell power allocation vector $\bar{p}^{*}{ }_{c}$ for any given $\bar{p}_{\text {var }}$. To efficiently solve $\left(\mathrm{IPC}_{c}\right)$ we use the methodology provided for the solution of problem (11) in [35]. Problem (11) in [35] is also a logarithmically transformed IPC problem under power constraints. Furthermore, the adopted utilities in our case [as defined in (2) and (7)] are subcases of the utilities considered in [35]. Therefore, for obtaining the solutions of (IPCs) we can directly utilize the gradient projection algorithm with constant step size proposed in [35] (Section IV), which is characterized by provable convergence and optimality attributes. We further refer to the latter algorithm as $\mathrm{IPC}_{c}$, which takes as input any $\bar{p}_{\text {var }}$ and provides as output the corresponding $\bar{p}^{*}{ }_{c}$.

\section{Deriving the Lagrange multiplier of an (IPCs)}

Prior to obtaining the solution of $(\mathrm{CM})$, it still remains to compute the unique optimal Lagrange multipliers $\left(\lambda_{c}^{*}\left(\bar{p}_{c, \text { var }}\right)\right)$ of all IPCs, associated with the corresponding constraints (14). The computation of those multipliers guarantees the distributed solution of $\mathrm{CM}$. To that end, the unique Lagrange multiplier of an $\left(\mathrm{ICP}_{c}\right)$ [the uniqueness results from (ICP) problems' convexity], given $\bar{p}^{*}{ }_{c}$ and any $\bar{p}_{c, \text { var }}$, can be calculated via the problem's Lagrangian, defined as

$$
\begin{aligned}
& L^{c}\left(\bar{p}_{c}, \bar{p}_{c, \text { var }}, \lambda_{c}\right) \equiv \sum_{j \in S_{c}} U_{j}\left(\gamma_{c, j}\left(p_{c, j}\right)\right) \\
& \quad+\lambda_{c}\left[\exp \left(p_{c, \text { var }}\right)-\sum_{j \in S_{c}} \exp \left(p_{c, j}\right)\right]
\end{aligned}
$$

Furthermore, taking into account the second-order necessary conditions for optimality [36], i.e., $\nabla_{\bar{p}_{c}} L^{c}\left(\bar{p}_{c}, \bar{p}_{c, \text { var }}, \lambda_{c}\right)$ $\mid \begin{aligned} & \bar{p}_{c}=\bar{p}_{c}^{*} \\ & \lambda_{c}=\lambda_{c}^{*}\end{aligned}=0$ and applying users' utility functions in (1), after some manipulation we get

$$
\begin{aligned}
& \nabla_{\bar{p}_{c}} L^{c}\left(\bar{p}_{c}, \bar{p}_{c, \text { var }}, \lambda_{c}\right) \mid \begin{array}{l}
\bar{p}_{c}=\bar{p}_{c}^{*} \\
\lambda_{c}=\lambda_{c}^{*}
\end{array} \\
& =0 \rightarrow \sum_{j \in S_{c}}\left\{\frac{\partial U_{j}\left(\gamma_{c, j}\left(p_{c, i}\right)\right)}{\partial p_{c, i}}-\lambda_{c}^{*} \exp \left(p_{c, i}\right)\right\} \mid \\
& \bar{p}_{c}=\bar{p}_{c}^{*} \rightarrow \lambda_{c}^{*}=\frac{\sum_{j \in S_{j}} k / k-G_{c, j} \exp \left(p_{c, j}^{*}\right)}{\exp \left(p_{c, \text { var }}\right)}
\end{aligned}
$$

where $k=\sum_{c \in C} G_{c, j} \exp \left(p_{c, \text { var }}\right)+n_{0}$

Concluding this section's analysis, let us underline that $\left(\mathrm{IPC}_{c}\right)$ algorithm for cell $c \in C$ takes as input any $\bar{p}_{\text {var }}$ and obtains (a) the corresponding $\bar{p}_{c}{ }_{c}$, as analyzed in "Treating IPC" section, as well as (b) the optimal objective value of $\left(\mathrm{ICP}_{\mathrm{c}}\right), U_{c} *\left(\bar{p}_{\text {var }}\right)$ for any given $\bar{p}_{\text {var }}$ (i.e., $\left.U_{c}^{*}\left(\bar{p}_{\text {var }}\right) \equiv \mathrm{U}_{c}\left(\bar{p}^{*}{ }_{c}, \bar{p}_{\text {var }}\right)=\sum_{j \in S_{c}} U_{j}\left(\gamma_{c, j}\left(p^{*}{ }_{c, j}\right)\right)\right)$, with a subgradient given by the multiplier $\lambda_{c}{ }^{*}$.

\section{Treating $\mathrm{CM}$ (the master problem)}

In line with the previous analysis, given the optimal intra-cell power vector $\bar{p}^{*} \forall c \in C$, the master CM problem can be defined as

$$
\begin{aligned}
& (\mathrm{CM}) \underset{\bar{p}_{\mathrm{var}}}{\operatorname{maximize}} \sum_{c \in C} U_{c}^{*}\left(\bar{p}_{\mathrm{var}}\right) \\
& \text { s.t. } \quad P_{c}^{\text {Min }} \exp \left(-p_{c, \mathrm{var}}\right) \leq 1 \quad \forall c \in C \\
& \left(P_{c}^{\text {Max }}\right)^{-1} \exp \left(p_{c, \mathrm{var}}\right) \leq 1 \quad \forall c \in C
\end{aligned}
$$

Problem constraints (18) and (19) can be further combined and rewritten in an equivalent but more flexible to treat form as

$$
\begin{aligned}
& \text { (CM) } \underset{\bar{p}_{\mathrm{var}}}{\operatorname{maximize}} \sum_{c \in C} U_{c}^{*}\left(\bar{p}_{\mathrm{var}}\right) \\
& \text { s.t. } \log \left(P_{c}^{\text {Min }}\right) \leq \mathrm{p}_{c, \mathrm{var}} \leq \log \left(P_{c}^{\text {Max }}\right) \quad \forall c \in C
\end{aligned}
$$

where cells' optimal power vectors $\bar{p}^{*}{ }_{c}$ are known, log $\left(P_{c}^{\mathrm{Max}}\right)$ and $\log \left(P_{c}^{\mathrm{Min}}\right)$ are constants and only the optimal transmit power vector of all cells' BSs $\bar{p}_{\text {var }}^{*}$ (i.e., cells' coverage) is left to be computed. 
Towards solving CM, we apply a subgradient method based on [34]. Our approach is mainly motivated by the following:

a. the gradient of the objective function of $\mathrm{CM}$ is hard to compute,

b. it is shown in $[33,34]$ that the subgradient of each $U_{c}^{*}\left(\bar{p}_{\text {var }}\right)$ is equal to the optimal Lagrange multiplier corresponding to the constraint (14) in $\left(\mathrm{ICP}_{c}\right)$.

Therefore, $\nabla c U_{c}^{*}\left(\bar{p}_{\mathrm{var}}\right)^{c}=\lambda_{c}^{*}\left(p_{c, \mathrm{var}}\right)$, which is computed in (16). Finally, the global subgradient is $\nabla$ $\bar{p}_{\text {var }} U_{c}^{*}\left(\bar{p}_{\mathrm{var}}\right)^{c}=\sum_{c \in C} \lambda_{c}^{*}\left(\bar{p}_{\mathrm{var}}\right)$.

The master primal problem (CM) can thus be solved using a subgradient method (denoted as CM algorithm) via updating cells' maximum power levels as shown next.

$$
\left[\begin{array}{l}
p_{0, \operatorname{var}}(t+1) \\
\cdots \\
p_{|C|-1, \operatorname{var}}(t+1)
\end{array}\right]=\left[\left[\begin{array}{l}
p_{0, \operatorname{var}}(t) \\
\cdots \\
p_{|C|-1, \operatorname{var}}(t)
\end{array}\right]+a(t)\left[\begin{array}{l}
\lambda_{0}^{*}\left(\bar{p}_{\mathrm{var}}(t)\right) \\
\ldots \\
\lambda_{|C|-1}^{*}\left(\bar{p}_{\mathrm{var}}(t)\right)
\end{array}\right]\right]_{P}
$$

where $t$ denotes algorithm's iterations, $a(t)$ is the subgradient step, and [.] ${ }_{P}$ denotes the projection onto the feasible convex set:

$$
\begin{aligned}
P \triangleq & \left\{\left(p_{0, \mathrm{var}}, \ldots, p_{|C|-1, \mathrm{var}}\right): \log \left(P_{c}^{\mathrm{min}}\right)\right. \\
& \left.\leq p_{c, \mathrm{var}} \leq \log \left(P_{c}^{\max }\right), \forall c \in C\right\} .
\end{aligned}
$$

Nicely enough this feasible set enjoys the property of naturally decomposing into a Cartesian product for each cells: $P=P_{0} \times P_{1} \times \cdots \times P_{|c|-1}$. Therefore, the predescribed subgradient update can be performed independently by each cell, simply with the knowledge of its correspondent (IPC) problem Lagrange multiplier $\lambda_{c}{ }^{*}$, which in turn is also independently computed and updated by each cell's $c\left(\mathrm{ICP}_{c}\right)$.

Therefore, all algorithms that constitute PCC, i.e., ICPs and $\mathrm{CM}$, are distributively solved by each system cell. The only required information that needs to be disseminated among the cells of the two-tier system is $\bar{p}_{\text {var }}(t)$ and $a(t)$, imposing minimal overhead over the system.

\section{Distributed PCC algorithm}

In this section, we analyze the operation and justify the designing attributes of the proposed PCC algorithm. Furthermore, it's converge to system's global optimal operation point is proved, while it's speed of convergence and the imposed signaling overhead are discussed.

\section{Design and operation}

Towards implementing the joint CM and power control algorithm, the cooperation of the ICPs and CM algorithms is required. Specifically, ICP algorithms, residing on every cell $c \in C$, are responsible for solving the corresponding intra-cell (macro or femto) power control and resource allocation problem. The CM algorithm in each femtocell dynamically updates cell's maximum transmission power, in line with (23), while the CM one at the macro base station guarantees the synchronization among all cells via gathering and distributing $\bar{p}_{\mathrm{var}}(t)$ and $a(t)$. It is important to note that both ICP and CM algorithms are located in each cell's base station and thus all information exchanged between them is local and does not impose any signaling overhead to the wireless medium.

The PCC algorithm responsible for achieving optimal $\mathrm{CM}$ and power control in a two-tier heterogeneous system can be summarized in Table 1 . Moreover, Figure 2 illustrates algorithm's overall operation, mainly focusing on the required disseminated information among the different algorithms, supporting our allegations on minimal signaling overhead.

\section{On addressing convergence and optimality}

In this section, we argue on the optimality and the convergence properties of the proposed PCC management

\begin{tabular}{|c|c|c|c|}
\hline \multicolumn{4}{|c|}{ Primal-dual algorithm to solve PCC (P3): } \\
\hline Initialization & Set $t=0$ and $\bar{p}_{\text {var }}(0)$ equal to a non-negative value. & & \\
\hline Step & Operation & Input & Output \\
\hline 1 & $\begin{array}{l}\text { Solve ICPs at each cell considering the specific users' utilities as well as the power vector } \\
\bar{p}_{\text {var }}(t) \text {, via the canonical dual algorithm in Section IV.A, deriving cell's optimal utility vector, } \\
\text { the normalized Lagrangian multiplier and users' power vector for the specific } \bar{p}_{\text {var }}(t) \text {. This } \\
\text { implies a iterative algorithm. }\end{array}$ & $\begin{array}{l}U_{j} \forall j \in S_{j} \\
\bar{P}_{\mathrm{var}}(t)\end{array}$ & $\begin{array}{l}\bar{U}_{c}^{*}\left(\bar{p}_{c}^{*}, \bar{p}_{\mathrm{var}}\right) \\
\lambda_{c}^{*}\left(\bar{p}_{\mathrm{var}}(t)\right) \\
\bar{p}_{c}^{*}(t) \forall c \in C\end{array}$ \\
\hline 2 & $\begin{array}{l}\text { Each cell } c \text { dynamically updates its maximum transmission power } p_{c, v a r}(t+1) \text { in accordance } \\
\text { to the gradient algorithm (23). This information is sent to the macro cell using existing } 3 G P P \\
\text { signaling. }\end{array}$ & $\begin{array}{l}\lambda_{c}^{*}\left(\bar{p}_{\mathrm{var}}(t)\right) \\
a(t)\end{array}$ & $\begin{array}{l}p_{c, \operatorname{var}}(t+1) \\
\forall c \in C\end{array}$ \\
\hline 3 & $\begin{array}{l}\text { Set } t \leftarrow t+1 \text {, disseminate } \bar{p}_{\text {var }}(t), a(t) \text { and go to step } 1 \text { (until satisfying } \\
\text { termination criterion). Upon convergence, optimal } C M \text { and power control vector }\left(\bar{p}_{c}^{*}, \bar{p}^{*}{ }_{\text {var }}\right) \\
\text { have been derived. }\end{array}$ & & $\left(\bar{p}_{c}^{*}, \bar{p}^{*}{ }_{\text {var }}\right)$ \\
\hline
\end{tabular}
algorithm.

\section{Table 1 PCC algorithm}




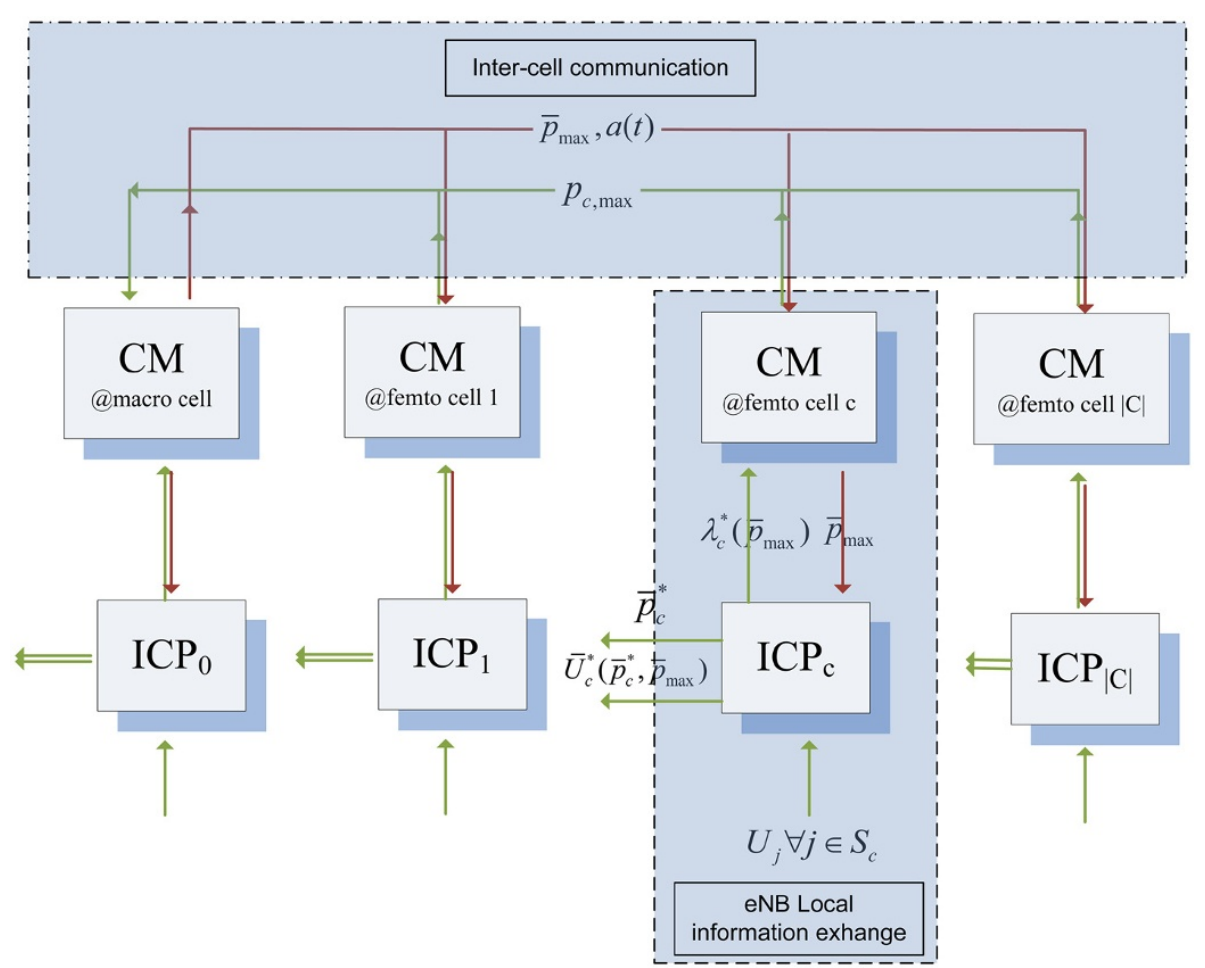

Figure 2 Power control and cells' coverage algorithm signaling.

Initially, we argue on the selected update step for the proposed subgradient method (22), which is a diminishing step size rule $a(t)$ with the following properties: $a(t)>0$, $\lim _{t \rightarrow \infty} a(t)=0$ and $\sum_{t=0}^{\infty} a(t)=\infty$. In accordance to [36], since $\mathbb{C}$ is compact and not empty, and CM is a convex optimization problem, then via a diminishing step size rule, for example $a(t)=\frac{t}{\beta+t}$ where $\beta>1$ is a fixed constant, the subgradient algorithm converges, i.e., $\bar{p}_{\text {var }}(t) \rightarrow \bar{p}_{\text {var }}^{*}$ as $t \rightarrow \infty$ and thus $\lim \sup \left\{\sum_{c \in C} U_{c}^{*}\left(\bar{p}_{\text {var }}\right)\right\} \rightarrow U_{\text {TOTAL }}^{*} \triangleq \sum_{c \in C} U_{c}^{*}\left(\bar{p}_{\text {var }}\right)$. In addition to that, since via $\left(\mathrm{ICP}_{c}\right)$ we can compute $\bar{p}^{*}\left(\bar{p}_{\text {var }}\right) \forall \bar{p}_{\text {var }}$ the convergence of $\bar{p}_{\text {var }}(t)$ to the global optimum implies the global optimality of $\bar{p}^{*}\left(\bar{p}_{\text {var }}^{*}\right)$. Therefore, we conclude that both variable vectors $\left(\bar{p}_{c}, \bar{p}_{\text {var }}\right)$ converge to system's global optimal values $\left(\bar{p}_{c}^{*}, \bar{p}^{*}\right.$ var $)$ leading to overall two-tier system throughput maximization.

\section{Discussions and design features}

- Synchronization: The existence of the diminishing step size $a(t)$ demands the synchronization among cells in the two-tier system when computing $\bar{p}_{\text {var }}(t+1)$. Thus, all cells are required to maintain the value of the current iteration, as well as cells' maximum power vector $\bar{p}_{\text {var }}(t)$. In other words, it is required to exchange the values of $a(t)$ and $p_{c \text {,var }}(t)$ per iteration $t$. This is feasible, especially considering the nature of the two tier system since (a) CDMA is slotted by nature, thus synchronization is inherent, and (b) the macrocell, as the overlay cell, can broadcast theses values using its broadcast channel, a common practice in 3GPP LTE [37].

- Signaling overhead: In line with the previous analysis, the imposed signaling overhead is minimal and comprises of only $|C|+1$ real numbers per iteration $\left[a(t)\right.$ and $\left.\bar{p}_{\text {var }}\right]$ which can easily be carried out via existing signaling.

- Convergence speed: Although the convergence to optimality is proven, the time of the convergence cannot easily be determined or even bounded. Practically, it depends on the tradeoff between accuracy and chosen time-complexity, which further depends on the termination threshold $\varepsilon$ we set, i.e., if $\left\|\bar{p}_{\text {var }}(t+1)-\bar{p}_{\text {var }}(t)\right\| \leq \varepsilon$ and $\varepsilon<a(t)$ then stop. However, as shown in the following section, the experimental results indicate that under all cases the algorithm converges in less than 30 iterations.

\section{Numerical results}

In this section, we provide indicative numerical results demonstrating the effectiveness of the proposed PCC management algorithm, as well as providing a proof of concept of its applicability in a two-tier cellular environment. The results reveal that PCC achieves significant performance gains under various femtocell/macrocell 
network deployment topologies, in terms of overall achieved utility-based performance (i.e., system throughput) and power consumption, compared to a power control (PC) policy that aims only at overall integrated system performance optimization (i.e., via enabling optimal IPC fall all cells) without $\mathrm{CM}$.

We consider a two-tier network assembled by one CDMA macrocell and $|C|-1$ CDMA underlay co-channel femtocells organized in clusters, as illustrated in Figures 3a and $4 \mathrm{a}$, towards simulating femtocells deployment over urban areas [38]. We assume that CDMA system's spreading bandwidth is $W=10 \mathrm{MHz}$, while the upper bound on macrocell and femtocells downlink transmission power due to physical limitations is set $P_{0}^{\max }=10 \mathrm{~W}$ and $P_{c}^{\max }=3 \mathrm{~W}$ for $c=1, \ldots,|C|-1$, respectively [38,39]. Macrocells' overall transmission power is fixed. Furthermore, each user is assigned a proper utility function of his achieved downlink rate in accordance to his received SINR as modeled in (2). In each scenario, various percentages of users' population distribution among the macrocell and the femtocell layers are considered. The percentage of total number of users associated with femtocells is denoted as FUP (i.e., femtocell users' percentage).

Initially, we consider a two-tier system with 14 femtocells assembling 2 clusters, as depicted in Figure 3a. System performance, in terms of (a) overall aggregated utility (i.e., throughput) increment, (b) macrocell's aggregated utility (i.e., throughput) increment, and (c) overall power consumption decrement under PCC compared to PC is illustrated in Figure $3 \mathrm{~b}-\mathrm{d}$, respectively, for increasing active (a)

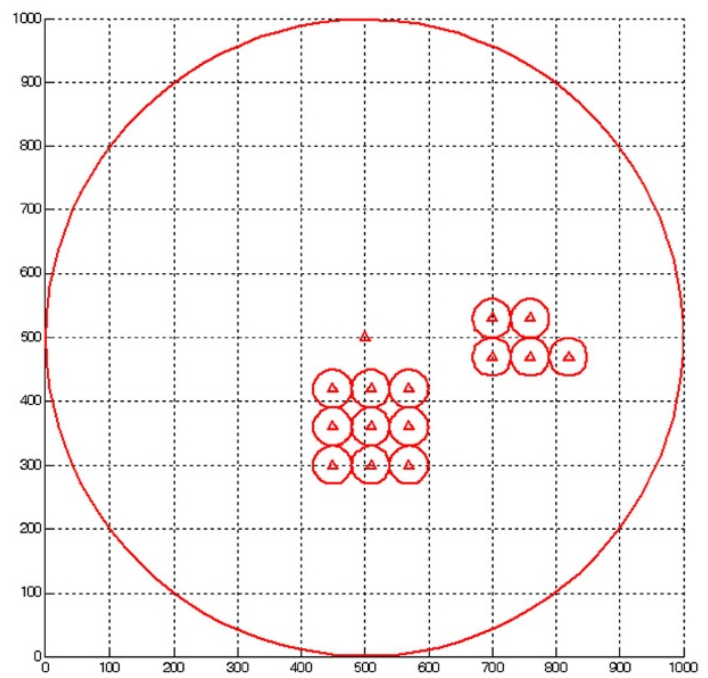

(c)

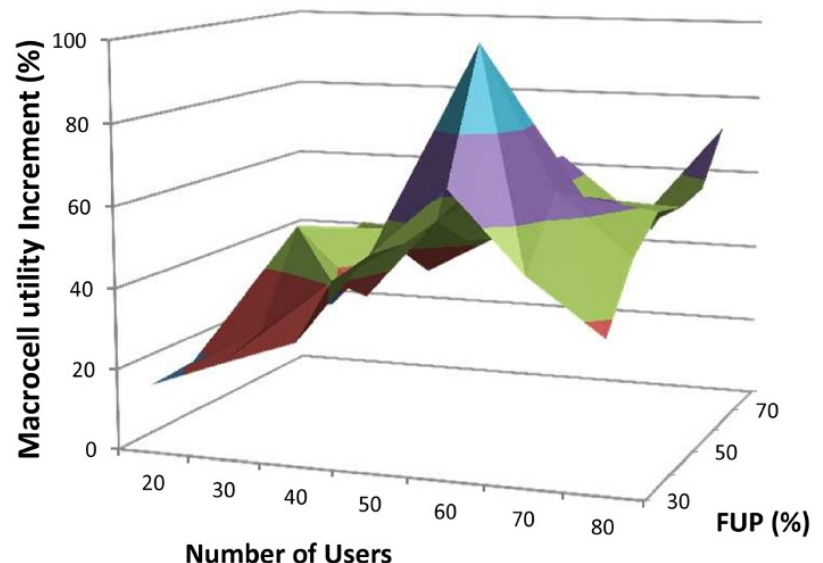

(b)

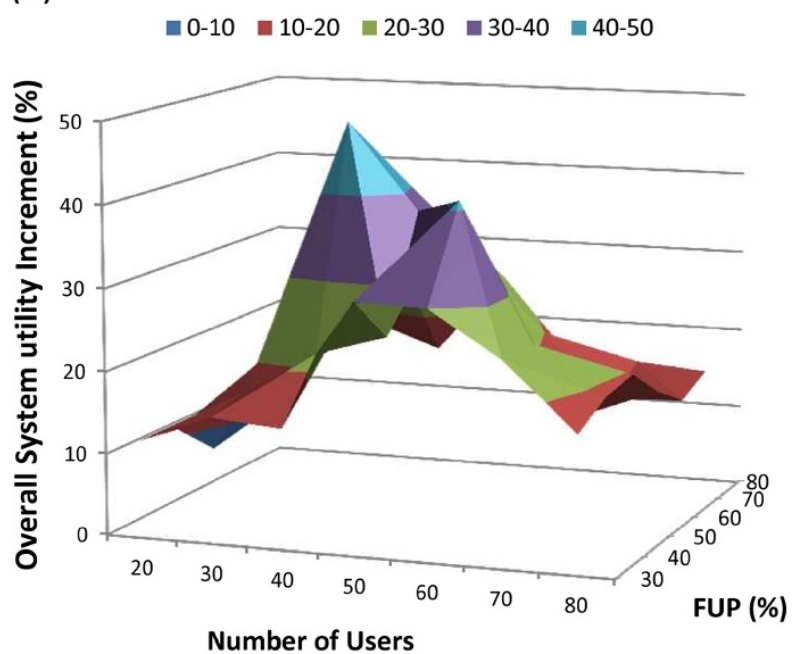

(d)

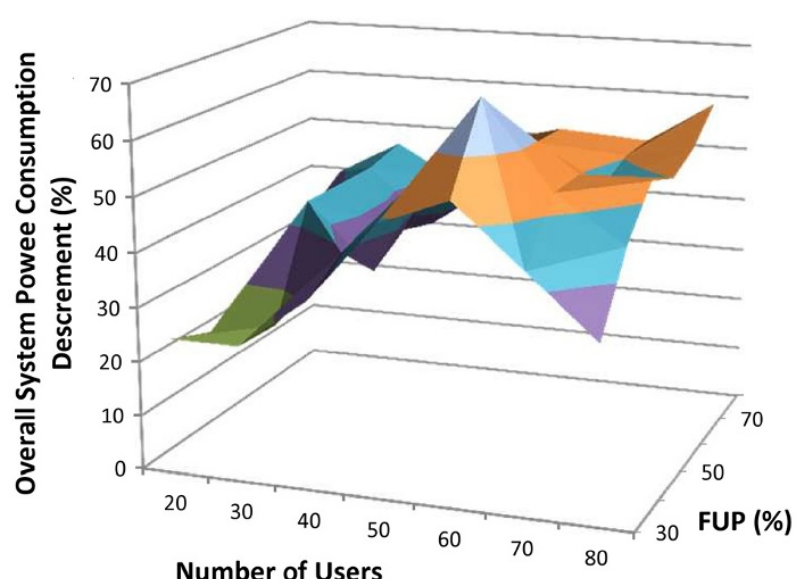

Figure 3 Experimental results for two active clusters. (a) A two-tier system with 14 femtocells assembling 2 clusters is considered. (b) System performance results on overall aggregated utility (i.e., throughput) increment, (c) macrocell's aggregated utility (i.e., throughput) increment, and (d) overall power consumption decrement under PCC compared to PC are illustrated. 

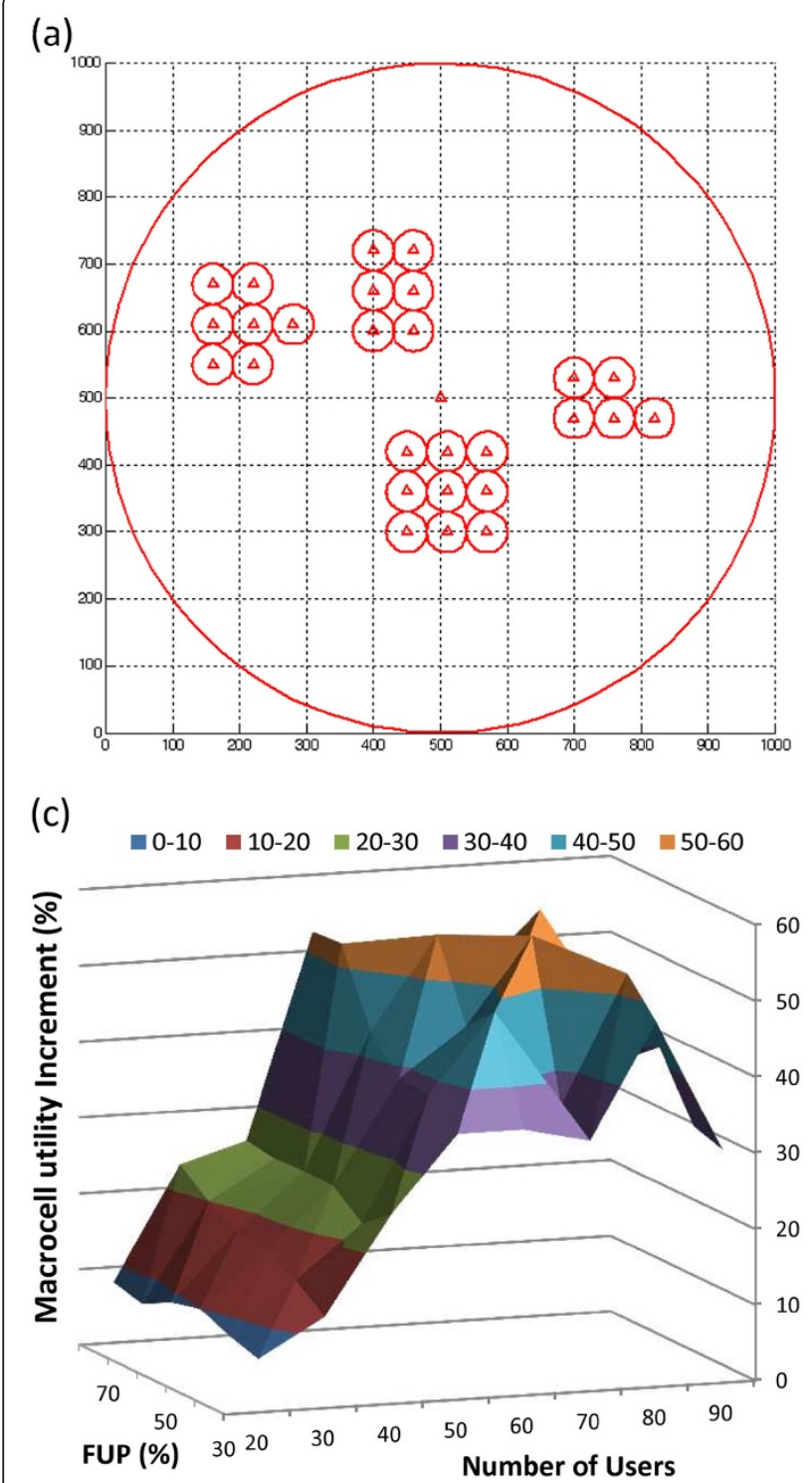

(b)

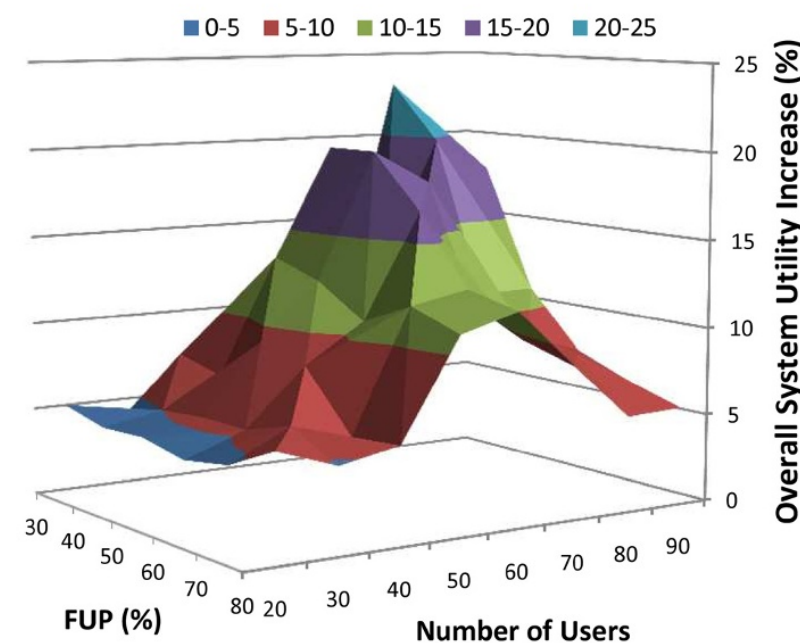

(d)

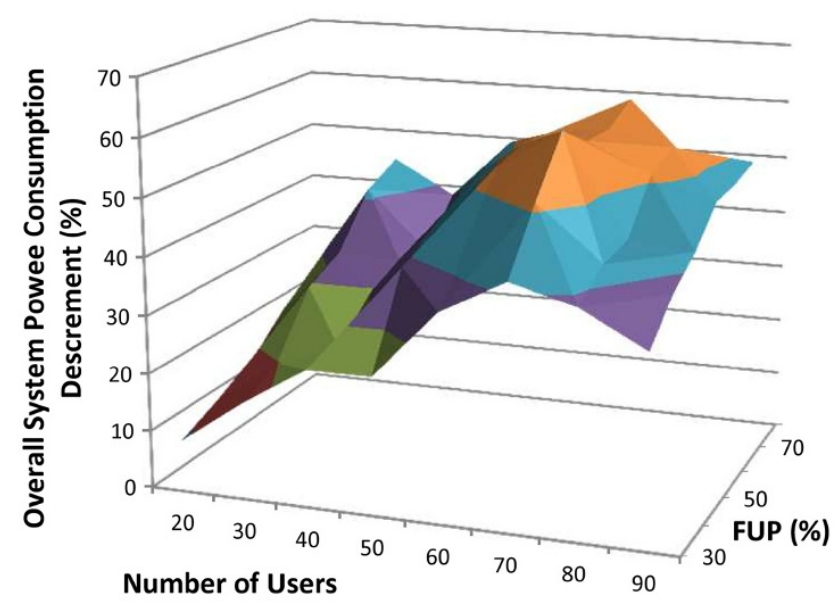

Figure 4 Experimental results for four active clusters. (a) A two-tier system with 27 femtocells assembling 4 clusters is considered. (b) System performance results on overall aggregated utility (i.e., throughput) increment, (c) macrocell's aggregated utility (i.e., throughput) increment, and (d) overall power consumption decrement under PCC compared to PC are illustrated.

users' population (i.e., $|J|=20,30, \ldots, 80$ ), arbitrarily placed in the system, and femtocell users' percentages (i.e., FUP $=30,40, \ldots, 80$ ).

The results show that under realistic (i.e., most expected $[38,39])$ user population distributions, i.e., number of femtocells (14), number of overall users (from 40 to 60) and femtocell users' percentage of 40-60\%, which corresponds to an average of 2 to 3 users per femtocell (most common case over a practical femtocell deployment), overall system utilization (i.e., two-tier system throughput) is increased up to $50 \%$ under the proposed PCC algorithm, as revealed in Figure $3 \mathrm{~b}$.
The main reason for attaining such a highly desirable system performance increase under $\mathrm{PCC}$ is related to the high improvement of overall macrocell users' performance, which in many cases exceeds $60 \%$, i.e., from 30 to $48 \mathrm{Mbps}$ excess achieved two-tier capacity in some scenarios (Figure 3c), as a result of PCC algorithm's optimal CM control in the two-tier network. Specifically under PCC, femtocells' coverage areas are optimally fine tuned, therefore their overall maximum power is decreased (while maintaining optimal intra-cell utility-based performance). This allows macrocell users to experience a highly reduced cross-tier inter-cell interference and thus, 
establish high throughput performance gains, compared to the case where only optimal PC is achieved.

The latter behavior is also confirmed by the results presented in Figure 3d, where the percentage of overall system power reduction under PCC is up to $70 \%$, with respect to the corresponding one under $\mathrm{PC}$ (i.e., from 55 to $17 \mathrm{~W})$. This is due to femtocells' efficient coverage control, under which femtocells' maximum downlink power allowed to be allocated to their corresponding attached users, is reduced up to the point where their utility-based performance remains high. This, not only avoids high femtocell resource over provisioning, but also leads to femtocells' coverage leakage reduction and thus, to overall two-tier interference mitigation.

Nevertheless, it should be pointed out that the benefits of joint PCC management under PCC are condensed, when moving at the boundaries of users' population distributions among the two tiers. Specifically, for very low or very large percentages of femtocell users (i.e., less than $30 \%$ or more than $70 \%$, corresponding to less than 2 or more than 5 users per femtocell on average), overall system utility-based performance improvement under PCC decreases, even though macrocell users still experience significant performance gains. This latter behavior is expected, since in the first case femtocells become underloaded (i.e., there are no active femtocell users to serve), therefore no CM can be performed, while on the second case, most femtocells are overloaded and thus, towards efficiently serving all attached users maintain high downlink transmission powers, resulting to high cross-tier interference.

The same patterns can be observed even if we increase the complexity of the system, i.e., adding two more clusters in the system as illustrated in Figure 4a-d. Specifically, reductions of up to $60 \%$ in the overall cumulative power consumption are observed, saving up to $50 \mathrm{~W}$, while the macrocell users enjoy up to $50 \%$ utility increase, implying a $15-\mathrm{Mbps}$ boost in their aggregated performance. Moreover, we should note that low values of performance gains observed do not imply that the algorithm performs poorly, but that the system is already close to its optimal state, leaving little room for improvement.

Finally, we also focus on the algorithmic attributes of the proposed PCC algorithm, and specifically on its rate of convergence, since the latter also affects both the signaling overhead imposed into the two-tier network, as well as its practicality and applicability. In Figure 5, the minimum, maximum, and average number of iterations required by the proposed PCC algorithm to achieve a stable system operational point (i.e., convergence of the proposed subgradient scheme) as a function of FUP averaged for all user populations are illustrated, in the case of two (blue lines) and four (red lines) active clusters scenarios. The results reveal that algorithm's convergence requires on average 15 iterations and no more than 30 iterations in all studied cases. Practically, the latter observation indicates that in the case of an HDR-CDMA system whose time-slot duration is 1.67 $\mathrm{ms}$, the required time for PCC to converge would not excide $50 \mathrm{~ms}$.

\section{Concluding remarks and future work}

An optimal joint PCC management framework for twotier CDMA macrocell/femtocell wireless networks in the presence of co-tier and cross-tier interference has been

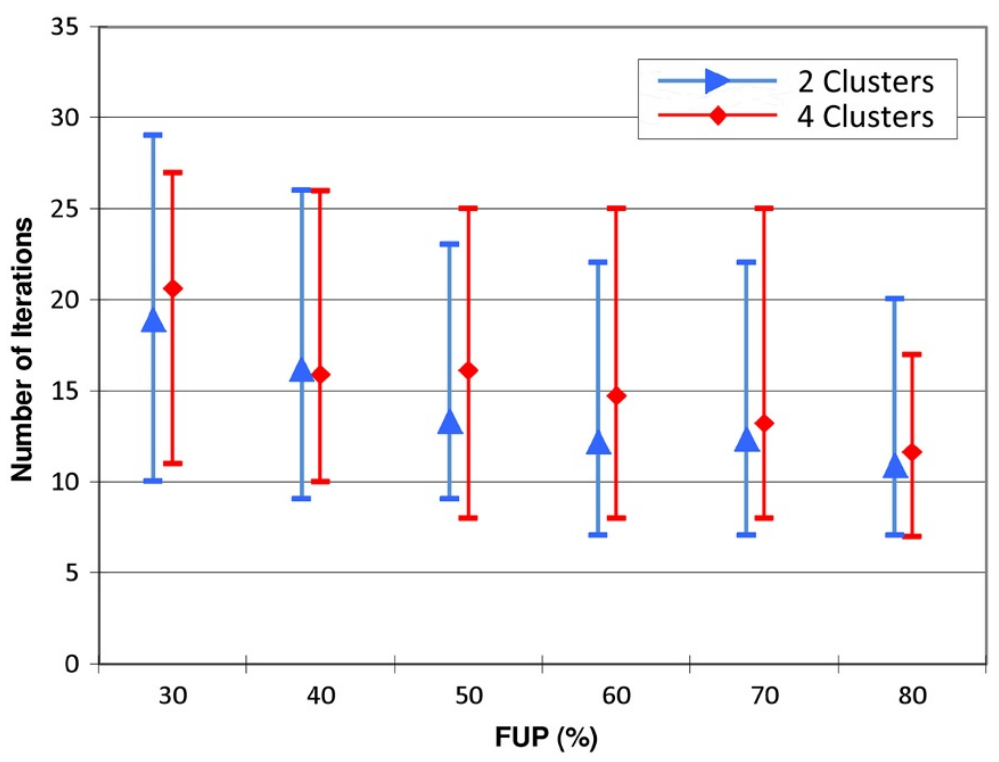

Figure 5 Number of iterations required for PCC algorithm's convergence. 
presented, aiming at maximizing users' downlink throughput performance, while minimizing cells' excess cumulative power consumption and thus, overall interference. Towards this goal, the integrated network is viewed as a global optimizer and the corresponding network-wide NUM problem is treated via a two-level primal-dual decomposition, allowing us to devise both optimal and distributed solution. A low complexity algorithm with provable convergence attributes was realized, that exploits cells' intra-cell resource allocation process using a subgradient Lagrangian scheme. Finally, initial numerical results are presented, demonstrating up to $60 \%$ improvement in both system's performance increase and overall power consumption savings.

It is important to note that even though the proposed two-level primal-dual decomposition architecture presented and evaluated in this article was applied on CDMA macrocell/femtocell wireless systems, it can be further extended accordingly to support several other wireless access schemes, given the proven separability and convexity of both the objective function and constraints. Thus, at the lower decomposition level, IPC problems will achieve optimal intra-cell resource allocation (e.g., for OFDM both transmit power and subcarrier vectors needs to be derived). At a higher level, a CM master problem will be responsible for dynamically determining optimal cells' coverage area towards network-wide performance optimality.

Moreover, the proposed framework assumes CA for femtocell users, meaning that only subscribed users can access the femtocell infrastructure. An interesting future direction would be the treatment of the PCC problem assuming Hybrid or Open access. This would add an extra binary cell assignment constraint to the overall problem formulation, amplifying its complexity and non-convex nature. Another interesting future extension would be the incorporation of (i) minimum SINR requirements for mobile users, towards minimum QoS-requirement incorporation or (ii) femtocells' maximum available bandwidth limitations, imposed by their DSL backbone architecture. A common direction towards addressing all these problems would be either a complex triple level primaldual-dual decomposition, or a sophisticated two-level dual decomposition.

\section{Appendix}

\section{Proof of Proposition 1}

Our proof is founded on (a) the monotonic property of a user's utility as a function of the achieved SINR and (b) on the nature of the two-tier system downlink SINR, for any given $\bar{P}_{\max }$. To that end, we follow and extend the thread of analysis in [23], Proposition 1, where similaroptimization problem is treated in the case of one cell power control only.
For any given feasible $\bar{P}_{\text {var }}$ and for any cell $w \in C$, let $\bar{P}=\left(\bar{P}_{0}, \ldots, \bar{P}_{w}, \ldots, \bar{P}_{|C|-1}\right)$ be a power allocation vector such that $\sum_{j \in S_{w}} P_{w, j}<P_{w, \text { var }}$. Let us underline that for the rest of the cells' power allocation no assumption is made (i.e., the following hold for any potential power allocation vector values for the rest of the cells in the system, within feasibility bounds, including the optimal one). It suffices to show that there always exists another power allocation $\bar{P}^{\prime}=\left(\bar{P}_{0}, \ldots, \bar{P}_{w}^{\prime}, \ldots, \bar{P}_{|C|-1}\right)$ such that $\sum_{j \in S_{w}} P_{w, j}^{\prime}=P_{w, v a r}$, that improves the intracell utilitybased power allocation.

If $\sum_{j \in S_{w}} P_{w, j}<P_{w, \mathrm{var}}$, then there always exists at least one $h>1$ such that $\sum_{j \in S_{w}} P_{w, j}<h \sum_{j \in S_{w}} P_{w, j}=P_{w, \text { var }}$ for any cell $c$ in the system. Thus, we can directly define $P_{w}^{\prime}$, $j=h \cdot P_{w, j} \forall j \in S_{w}$ and from (1) we have:

$$
\begin{aligned}
\gamma_{w, j}\left(\bar{P}^{\prime}\right)= & \frac{G_{w, j} P_{w, j}^{\prime}}{G_{w, j} \sum_{j^{\prime} \neq j}^{j^{\prime} \in S_{w}} P_{w, j^{\prime}}^{\prime}+\sum_{c^{\prime} \neq w}^{c^{\prime} \in C} G_{c^{\prime}, j}\left(\sum_{k \in S_{c^{\prime}}} P_{c^{\prime}, k}\right)+n_{0}} \\
= & \frac{h \cdot G_{w, j} P_{w, j}}{h \cdot G_{w, j} \sum_{j^{\prime} \neq j}^{j^{\prime} \in S_{w}} P_{w, j^{\prime}}+\sum_{c^{\prime} \neq w}^{c^{\prime} \in C} G_{c^{\prime}, j}\left(\sum_{k \in S_{c^{\prime}}} P_{c^{\prime}, k}\right)+n_{0}} \\
& >\frac{h \cdot G_{w, j} P_{w, j}}{h \cdot G_{w, j} \sum_{j^{\prime} \neq j}^{j^{\prime} \in S_{w}} P_{w, j^{\prime}}+h \cdot \sum_{c^{\prime} \neq w}^{c^{\prime} \in C} G_{c^{\prime}, j}\left(\sum_{k \in S_{c^{\prime}}} P_{c^{\prime}, k}\right)+h \cdot n_{0}} \\
& =\gamma_{w, j}(\bar{P})
\end{aligned}
$$

Thus, $\forall j \in S_{w}$ it holds that $U_{j}\left(\gamma_{w, j}\left(\bar{P}^{\prime}\right)\right)>U_{j}\left(\gamma_{w, j}(\bar{P})\right)$ due to the monotonicity of $U$ as a function of $\gamma$, and thus, $\sum_{j \in S_{w}} U_{j}\left(\gamma_{c, j}\left(\bar{P}^{\prime}\right)\right)>\sum_{j \in S_{w}} U_{j}\left(\gamma_{c, j}(\bar{P})\right)$.

Therefore, each cell must transmit at its maximum feasible power $\bar{P}_{w \text {,var }}$ regardless of state of its neighbors, in order to achieve maximum intracell utility-based performance. Generalizing the above, and towards maximizing the cumulative system utility, every cell must transmit at its own maximum feasible power, or in reverse, vector $\bar{P}_{\text {var }}=\left(\bar{P}_{1, \text { var }}, \ldots, \bar{P}_{w, \text { var }}, \ldots, \bar{P}_{|C|-1, \text { var }}\right)$ guarantees maximum total two-tier system cumulative utility. This completes the proof.

\section{Competing interests}

The authors declare that they have no competing interests.

Received: 24 November 2011 Accepted: 23 September 2012 Published: 30 October 2012

\section{References}

1. V Chandrasekhar, JG Andrews, Femtocell networks: a survey. IEEE Commun. Mag. 46(9), 59-67 (2008)

2. D Calin, H Claussen, H Uzunalioglu, On femto deployment architectures and macrocell offloading benefits in joint macro-femto deployments. IEEE Commun. Mag. 48(1), 26-32 (2010)

3. KS FMhiri, B Reguiga, R Bouallegue, G Pujolle, A power management algorithm for green femtocell networks, in Proceedings of the 10th IFIP Annual Mediterranean Ad Hoc Networking Workshop (Med-Hoc-Net) (Sicily, Italy, 2011), pp. 45-49 
4. P Mach, Z Becvar, QoS-guaranteed power control mechanism based on the frame utilization for femtocells. EURASIP J. Wirel. Commun. Netw. (2011). doi:10.1155/2011/259253

5. H Jo, C Mun, J Moon, J Yook, Self-optimized coverage coordination in femtocell networks. IEEE Trans. Wirel. Commun. 9(10), 2977-2982 (2010)

6. H Jo, C Mun, J Moon, J Yook, Interference mitigation using uplink power control for two-tier femtocell networks. IEEE Trans. Wirel. Commun. 8(10), 4906-4910 (2009)

7. H Claussen, LTW Ho, LG Samuel, Self-optimization of coverage for femtocell deployments, in Proceedings of the Wireless Telecommunications Symposium, ed. by (Pomona, 2008), pp. 278-285

8. SY Choi, T Lee, MY Chung, H Choo, Adaptive coverage adjustment for femtocell management in a residential scenario, in Proceedings of the APNOMS, ed. by (Jeju, Korea, 2009), pp. 221-230

9. I Ashraf, H Claussen, LTW Ho, Distributed radio coverage optimization in enterprise femtocell networks, in Proceedings of the 2010 IEEE International Conference on Communications (ICC), ed. by (Cape Town, South Africa, 2010), pp. 1-6

10. Z Yalin, L Yang Yang, ES Sousa, Z Qinyu, Pilot power minimization in HSDPA femtocells, in Proceedings of the 2010 IEEE Global Telecom. Conference, ed. by (Miami, Florida, 2010), pp. 1-5

11. M Morita, Y Matsunaga, K Hamabe, Adaptive power level setting of femtocell base stations for mitigating interference with macrocells, in Proceedings of the 2010 IEEE 72nd Vehicular Technology Conference Fall (VTC 2010-Fall), ed. by (Ottawa, Canada, 2010), pp. 1-5

12. K Min-Sung, J Hui Won, FA Tobagi, Cross-tier interference mitigation for two-tier OFDMA femtocell networks with limited macrocell information, in Proceedings of the 2010 IEEE Global Telecommunications Conference, GLOBECOM, ed. by (Miami, Florida, 2010), pp. 1-5

13. Y Shu-ping, S Talwar, N Himayat, K Johnsson, Power control based interference mitigation in multi-tier networks, in Proceedings of 2010 IEEE GLOBECOM (FEMnet Workshop), ed. by (Miami, Florida, 2010), pp. 701-705

14. L Kisong, J Ohyun, DH Cho, Cooperative resource allocation for guaranteeing intercell fairness in femtocell networks. IEEE Commu. Lett. 15(2), 214-216 (2011)

15. T Akbudak, A Czylwik, Distributed power control and scheduling for decentralized OFDMA networks, in Proceedings of the 2010 Int. ITG Workshop on Smart Antennas, ed. by (Bremen, Germany, 2010), pp. 59-65

16. X Li, Q Lijun, D Kataria, Downlink power control in co-channel macrocell femtocell overlay, in Proceedings of the 43rd Annual Conf. on Inf. Sciences and Systems, ed. by (Baltimore, Maryland, 2009), pp. 383-388

17. H Kwanghun, C Youngkyu, K Dongmyoung, N Minsoo, C Sunghyun, H Kiyoung, Optimization of femtocell network configuration under interference constraints, in Proceedings of the WiOPT, ed. by (Seoul, Korea, 2009), pp. 1-7

18. T Chee Wei, Optimal power control in rayleigh-fading heterogeneous networks, in Proceedings of INFOCOM, ed. by (Shanghai, China, 2011), pp. $2552-2560$

19. V Chandrasekhar, JG Andrews, T Muharemovic, S Zukang, A Gatherer, Power control in two-tier femtocell networks. IEEE Trans. Wirel. Commun. 8(8), 4316-4328 (2009)

20. Z Jianmin, Z Zhaoyang, W Kedi, H Aiping, Optimal distributed subchannel, rate and power allocation algorithm in OFDM-based two-tier femtocell networks, in Proceedings of 2010 IEEE 71st Vehicular Technology Conference (VTC 2010-Spring), ed. by (Taipei, Taiwan, 2010), pp. 1-5

21. JH Yun, KG Shin, Adaptive interference management of OFDMA femtocells for co-channel deployment. IEEE J. Sel. Areas Commun. 29(6), 1225-1241 (2011)

22. A Ganti, TE Klein, M Haner, Base station assignment and power control algorithms for data users in a wireless multiaccess framework. IEEE Trans. Wirel. Commun. 5(9), 2493-2503 (2006)

23. J Lee, R Mazumdar, N Shroff, Joint resource allocation and base-station assignment for the downlink in CDMA networks. IEEE/ACM Trans. Netw. 14(1), 1-14 (2006)

24. P Hande, S Rangan, M Chiang, X Wu, Distributed uplink power control for optimal sir assignment in cellular data networks. IEEE/ACM Trans. Netw. 16(6), 1420-1433 (2008)

25. M Chiang, Balancing transport and physical layers in wireless multihop networks: jointly optimal congestion control and power control. IEEE J. Sel. Areas Commun. 23(1), 104-116 (2005)
26. A Goldsmith, Wireless Communications (Cambridge University Press, Cambridge, UK, 2004)

27. M Chiang, P Hande, T Lan, CW Tan, Power control in wireless cellular networks. Found. Trends Netw. 2(4), 381-533 (2008)

28. N Gatsis, A Marques, G Giannakis, Power control for cooperative dynamic spectrum access networks with diverse QoS constraints. IEEE Trans. Commun. 58(3), 933-944 (2010)

29. M Chiang, Nonconvex optimization of communication systems, in Advances in Mechanics and Mathematics Special Volume on Strang's 70th Birthday, ed. by D Gao, H Sherali (Springer, New York, 2007), p. 137

30. D Julian, M Chiang, D O'Neill, S Boyd, QoS and fairness constrained convex optimization of resource allocation for wireless cellular and ad hoc networks, in Proceedings of the IEEE Twenty-First Annual Joint Conf. of the IEEE Computer and Com. Societies, INFOCOM, ed. by, 2nd edn. (New York, 2002), pp. 477-486

31. S Boyd, L Vandenberghe, Convex Optimization (Cambridge University Press, Cambridge, UK, 2004)

32. M Chiang, S Boyd, Geometric programming duals of channel capacity and rate distortion. IEEE Trans. Inf. Theory 50(2), 245-258 (2004)

33. M Chiang, SH Low, AR Calderbank, JC Doyle, Layering as optimization decomposition: a mathematical theory of network architectures. IEEE Proc 95(1), 255-312 (2007)

34. DP Palomar, M Chiang, A tutorial on decomposition methods for network utility maximization. IEEE J. Sel. Areas Commun. 24(8), 1439-1451 (2006)

35. S Stanczak, M Wiczanowski, H Boche, Distributed utility-based power control: objectives and algorithms. IEEE Trans. Signal Process. 55(10) 5058-5068 (2007)

36. DP Bertsekas, A Nedi'c, A Ozdaglar, Convex Analysis and Optimization (Athena Scientific, Belmont, MA, 2003)

37. 3GPP TSG SA, 3GPP TS 23.402 V9.2.0, Architecture enhancements for non3GPP accesses. Release 9

38. 3GPP TSG RAN WG4 R4-092042, Simulation Assumptions and Parameters for FDD HeNB RF Requirements. (2008)

39. 3GPP TS 36.213 V 8.8.0, Evolved Universal Terrestrial Radio Access (E-UTRA) Physical Layer Procedures. (2009). Release 8

doi:10.1186/1687-1499-2012-329

Cite this article as: Aristomenopoulos et al:: Optimal power control and coverage management in two-tier femtocell networks. EURASIP Journal on Wireless Communications

and Networking 2012 2012:329.

\section{Submit your manuscript to a SpringerOpen ${ }^{\circ}$ journal and benefit from:}

- Convenient online submission

- Rigorous peer review

- Immediate publication on acceptance

- Open access: articles freely available online

- High visibility within the field

- Retaining the copyright to your article

Submit your next manuscript at $>$ springeropen.com 